\title{
A SYSTEMATIC IMAGE COMPRESSION IN THE COMBINATION OF LINEAR VECTOR QUANTISATION AND DISCRETE WAVELET TRANSFORM
}

\author{
K. Kalaivani ${ }^{1}$, C. Thirumaraiselvi ${ }^{2}$ \\ ${ }^{1}$ PG Student, Sri Krishna College of Engg \& Tech, Coimbatore \\ ${ }^{2}$ Associate Professor, Sri Krishna College of Engg \& Tech, Coimbatore
}

\begin{abstract}
As the use of digital image is increasing day by day, and the amount of data required for an acceptable quality image is high, there begins a high necessity for image compression. Vector quantisation $(V Q)$ is a novel technique for image compression. VQ is a lossy compression scheme, used to compress image both in spatial domain \& frequency domain. One of the major disadvantages is high encoding time \& complexity. In spite of these disadvantages it is highly preferred due to its advantages like high reconstruction quality at low coding rates and rapid decoding. Thus in order to reduce the high encoding time we go for the use of neural network. There are various types of neural networks are available. The proposed algorithm uses the most effective and simple methods like self organizing maps and linear vector quantization together with the discrete wavelet transform in order to reduce the loss of information during compression and their results are compared.
\end{abstract}

Keywords- Image compression, Neural network, Self organising maps, Linear vector quantization, discrete wavelet transform.

\section{INTRODUCTION}

A Common characteristic of most images is that the neighbouring pixels are correlated and therefore contain redundant information. The foremost task then is to find less correlated representation of the Image. Two fundamental components of compression are redundancy and irrelevancy reduction. Redundancy reduction aims at removing duplication from the signal source (image/video).Irrelevancy reduction omits parts of the signal that will not be noticed by the signal receiver namely Human Visual System(HVS).In general three types of redundancy can be identified as (i)Spatial Redundancy, (ii)Spectral redundancy, (iii) Temporal redundancy .

Image compression research aims at reducing the number of bits needed to represent an image by removing the spatial and spectral redundancies as much as possible. In many different fields, digitized images are replacing conventional analog images as photograph or x-rays. The volume of data required to describe such images greatly slow transmission and makes storage prohibitively costly. The information contained in images must, therefore, be compressed by extracting only visible elements, which are then encoded. The quantity of data involved is thus reduced substantially. The fundamental goal of image compression is to reduce the bit rate for transmission or storage while maintaining an acceptable fidelity or image quality.

One of the most successful applications of wavelet methods is transform-based image compression. A Discrete wavelets transform is the combination of the low pass and high pass filtering in a spectral decomposition of signals along with a very fast implementation. There are various reasons for choosing discrete wavelets transforms. They have extremely fast implementation, weighting factor. Their reciprocal can be implemented using only integer addition and bit shifts, which are extremely fast operation. The overlapping nature of the wavelet transform alleviates blocking artifacts, while the multiresolution character of the wavelet decomposition leads to superior energy compaction and perceptual quality of the decompressed image. Furthermore, the multiresolution transform domain means that wavelet compression methods degrade much more gracefully than block-DCT methods as the compression ratio increases. Wavelet-based coding [1] provides substantial improvements in picture quality at higher compression ratios.

Vector quantization is one of the commonly used techniques for data compression. A vector quantizer maps $\mathrm{k}$-dimensional vectors in the vector space $R_{k}[2]$ into a finite set of vectors $Y$ $=\{Y i: i=1,2 \ldots N\}$ each vector Yi is called a code vector or a code word, and the set of all the code words is called a 
codebook. The wavelet coefficients obtained at the wavelet decomposition level are converted into blocks. Then by applying vector quantization technique the blocks are converted into VQ code vectors. In the encoding stage, the VQ code vectors are compared with every SOFM code vectors in the initial codebook using Euclidean distance formula. Euclidean distance is computed as follows:

$$
D\left(x_{,} w_{i}\right)=\sqrt{\sum_{j=1}^{k}\left(t_{j}-w_{i j}\right)^{2}}
$$

The VQ code vectors found to be the closest in distance given by Eq.(1) from the input vector to the vector block is declared as the winning VQ code vectors. The indices of the winning VQ code. Vectors will be stored. These indices will be passed through a channel to the decoder.

Self-Organizing Map based neural network technique is used for initial codebook generation. SOM is a clustering technique having several desirable features and consequently it has attracted the attention of researchers in the field of vector quantization [3] [4]. The learning scheme of self-organizing map is based on Least Mean Square (LMS) [5] algorithm. In LMS algorithm the weights of the neurons are modified 'On the fly' for each input vector. SOM will generate more code vectors for the high density region.

Kohonen's learning vector quantization algorithm (LVQ) is a supervised variant of the self-organizing map algorithm (SOM) that can be used for labelled input data. Both SOM and LVQ are based on neurons representing prototype vectors and use a nearest neighbour approach for clustering and classifying data. So, they are neural network approaches particularly useful for non-linear separation problems. In LVQ labels associated with input data are used for training. The learning process tends to perform the vector quantization starting with the definition of decision regions and repeatedly reposting the boundary to improve the quality of the classifier.

\section{EXISTING METHODOLOGIES}

Shapiro [6] first introduced the notation of embedded coding called as Embedded Zero tree Wavelet coding (EZW) which yields output of low psycho visual quality image. Lewis and Knowles [7] constructed a wavelet function which uses the piecewise constant function. The wavelet function used by Lewis failed to produce image without blocking effects. Daubechies [2] introduced a family of compactly supported orthogonal wavelet systems with fixed regularity. The wavelet method proposed by Daubechies yields output with PSNR value ranges from $22 \mathrm{~dB}$ to $36 \mathrm{~dB}$ for various wavelet coefficients. Mallat [8] proposed the theory of multi resolution wavelet analysis known as Mallat algorithm which reduces the dimensionality with higher computational load. Pavlidis [9] introduced the scheme of polynomial surface fitting for modifying the code vectors generated by SOFM which failed to reduce the blockiness and dimensionality of the reconstructed image. Lloyd [10] constructed an algorithm for incremental update through competitive learning with higher loss of data.

Image compression based on the combination of wavelet and vector quantisation is done [11]. Wavelet compression along with the comparison after applying its seven families is worked by Jain \& Jain [12]. Implementation of $\mathrm{C}$ means clustering for codebook designing using fuzzy over wavelet tree coefficients [13]. Image compression using various wavelet approximations are proposed by DeVore et al [14].

Various applications of image processing use the combined effects of SOM and Wavelets. Image compression can be implemented by applying DWT over an image and SOM is applied to its result [15]. Combination of DCT, DWT and SOFM is also implemented, where SOFM is used to generate codebook [16]. Compression in the combination of SPIHT and SOFM has been implemented by Rawat and Meher [17]. Dandawate et al introduced a method of codebook generation for vector quantisation with SOFM and DWT [18].

\section{PROPOSED WORK}

In the proposed method, we are using a combination of artificial neural network and Discrete wavelet transform in order to perform the compression. Type of the neural network used differs from the existing methods. Here linear vector quantisation has been used to train the input vectors. Input image is splitted into non-overlapping blocks. Each block is converted into one dimensional vector, which is given as the input to the linear vector quantisation. As a result we get the trained weight matrix and Indexes were obtained. The trained output is given as the input to the discrete wavelet transform. We get the low low, low high, high low and high level components. The low low level components are encoded and stored and other components were left.

In order to reconstruct the image, we process in inverse. We first decode the stored low level components. The next step is to perform the inverse discrete wavelet transform. We keep the low components as it is and all other components like low high, high low and high components as zero and perform the inverse discrete wavelet transform. We then map the output of the inverse discrete wavelet transform with the indexes obtained as the result of linear vector quantisation. Now we have the output in the form of vectors. Thus in order reconstruct the image, the vectors are converted back into nonoverlapping blocks. Thus the reconstructed image is obtained. 
The flowchart for the above process is depicted as:

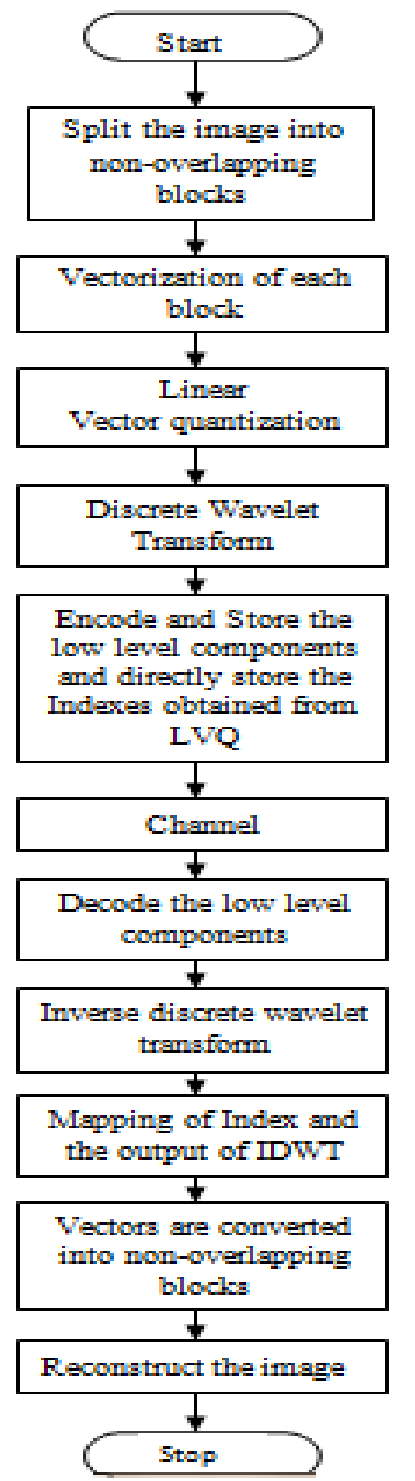

\section{RESULTS AND DISCUSSION}

Table 1: Comparison of PSNR values of various images with SOM and LVQ.

\begin{tabular}{|l|l|l|}
\hline \multirow{2}{*}{ IMAGE } & \multicolumn{2}{|l|}{ PSNR(dB) } \\
\cline { 2 - 3 } & SOM & LVQ \\
\hline Rice.png & 25.43 & 27.73 \\
\hline Peppers.png & 22.18 & 23.98 \\
\hline Coin.png & 24.44 & 26.24 \\
\hline Cameraman.bmp & 27.31 & 30.91 \\
\hline lena.bmp & 26.80 & 28.45 \\
\hline
\end{tabular}

Table 2: Comparison of MSE value of various images with SOM and LVQ

\begin{tabular}{|l|l|l|}
\hline \multirow{2}{*}{ IMAGE } & \multicolumn{2}{|l|}{ MSE } \\
\cline { 2 - 3 } & SOM & LVQ \\
\hline Rice.png & 352.7238 & 289.3657 \\
\hline Peppers.png & 537.7648 & 432.4961 \\
\hline Coin.png & 478.2030 & 392.0730 \\
\hline Cameraman.bmp & 163.9787 & 117.6012 \\
\hline lena.bmp & 275.6821 & 198.5149 \\
\hline
\end{tabular}

While comparing the results we get the better peak signal to noise ratio and better mean square error values. Thus the image is compressed in a better manner using linear vector quantisation than that of using self organising maps.

\section{CONCLUSIONS}

In this paper we have introduced a new method for image compression which uses artificial neural network and transform coding together. Here the type of artificial neural network used is linear vector quantisation and the type of transform coding used is discrete wavelet transform. In the algorithm an efficient codebook is obtained using linear vector quantisation. While the discrete wavelet transforms blocks the artifacts. The algorithm is very simple and computationally less complex. This linear vector quantisation performs better than many competitive networks like self organising maps. The proposed algorithm achieves high peak signal to noise ratio and reduced mean square error than many of the existing technologies. Here both the texture informations and the edge informations are preserved.

\section{REFERENCES}

[1]. Volkmer, H.,Onthe regularity of wavelets, IEEE Trans. on Information Theory,38, 872-876, 1992.

[2]. R.C. Gonzalez and R.E. Woods, "Digital Image Processing", Addison Wesley, 2007.

[3]. N. M. Nasrabadi and Y. Feng, "Vector Quantization of Images based upon the Kohonen Self-Organizing Feature Maps", Proceedings of International Conference on Neural Networks, Vol. 1, pp. 101-108, 1988.

[4]. E. Yair, K. Zager and A. Gersho,"Competitive learning and soft competition for vector Quantizer design", IEEE Transactions on Signal Processing, Vol. 40, No. 2, pp. 294309, 1992.

[5]. C. Amerijckx, M. Verleysen, P. Thissen and J D. Legat, "Image Compression by self organized Kohonen Map", IEEE Transactions on Neural Networks, Vol. 9, No. 3, pp. 503-507, 1998

[6]. Shapiro J. M, "Embedded Image Coding using Zero Trees of Wavelet Coefficients", IEEE Transactions on Signal Processing, Vol. 41, No. 12, pp. 3445-3462, 1993. 
[7]. A.S. Lewis and G. Knowles, "Image Compression using the 2-D Wavelet transform", IEEE Transactions on Image Processing, Vol. 1, No. 2, pp. 244-250, 1992.

[8]. S. Mallat, "A Theory for Multi Resolution Signal Decomposition: the Wavelet representation", IEEE Transactions on Pattern Analysis and Machine Intelligence, Vol. 11, No. 7, pp. 674-693, 1989.

[9]. T. Pavlidis, "Algorithms for Graphics and Image Compression", Springer- Verlag, 1982.

[10]. S. P. Lloyd, "Least Squares Quantization in PCM", IEEE Transactions on Information Theory, Vol. 28, No. 2, pp. 129137, 1982.

[11]. Karayiannis NB, Pin-I.Pai, Nicholas Zervos ,'Image compression based on fuzzy algorithm for learning vector quantization and wavelet image decomposition", IEEE Transaction on image processing,1998, vol 7(8), pp 1223 1230.

[12]. Jain YK, Jain Sanjeev," Performance evaluation of wavelets for image compression", Asian Journal of Information Technology,2006, 5(10):1104-1112.

[13]. Wang Xizhao, Wang Yadong, Wang Lijuan, "Improving fuzzy c-means clustering based on feature-weight learning", Pattern Recognisation Letters, 2004, 25(10):1123-1132.

[14]. DeVore RA, Jawerth Bjorne, Lucier Bradley J "Image compression through wavelet transform coding", IEEE Transaction Information Theory, 1992, 8(2):719-746.

[15]. Chatellier C, Boeglen H, Perrine C, Olivier C, Haeberle $\mathrm{O}$, "A robust joint source channel coding scheme for image transmission over the ionospheric channel.",Signal Processing Image Communication,2007, 22:543-556.

[16]. Immanuel Pandian S, Alex Anitha J," A neural network approach for color image compression in transform domain", Int Journal Recent Trends Engineering, 2009, 2(2):152-154.

[17]. Rawat CD, Meher Sukadev," A hybrid coding scheme combining SPIHT and SOFM based vector quantization for effectual image compression”, European Journal of Scientific Research, 2009, 38(3):425-440.

[18]. Dandawate YH, Jadhav TR, Chitra AV, Joshi MA, ” Neuro-Wavelet based vector quantizer design for image compression", Indian Journal of Science and Technology,2009, 2(10):56-61. 